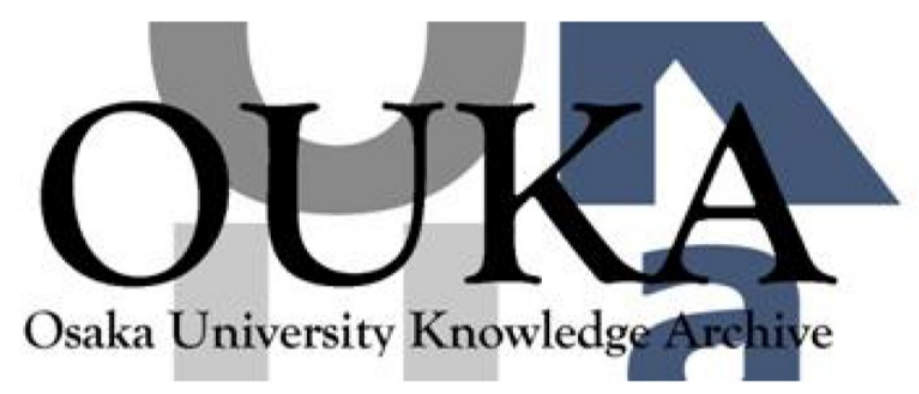

\begin{tabular}{|c|l|}
\hline Title & $\begin{array}{l}\text { Modulated photocarrier grating technique for } \\
\text { diffusion length measurement in amorphous } \\
\text { semiconductors }\end{array}$ \\
\hline Author(s) & Hattori, K.; Koji, Y.; Fukuda, S. et al. \\
\hline Citation & Journal of Applied Physics. 73(8) p. 3846-p. 3851 \\
\hline Issue Date & $1993-04-15$ \\
\hline oaire:version & VoR \\
\hline URL & https://hdl. handle. net/11094/3183 \\
\hline rights & \\
\hline Note & \\
\hline
\end{tabular}

Osaka University Knowledge Archive : OUKA

https://ir. Library. osaka-u. ac. jp/

Osaka University 


\title{
Modulated photocarrier grating technique for diffusion length measurement in amorphous semiconductors
}

\author{
K. Hattori, Y. Koji; S. Fukuda, W. Ma, H. Okamoto, and Y. Hamakawa \\ Faculty of Engincering Science, Osaka University, Toyonaka, Osaka 560, Japan
}

(Received 25 September 1992; accepted for publication 21 December 1992)

\begin{abstract}
This article presents a method for investigating the diffusion of photocarriers in semiconductors by the analysis of phase shift between a temporally modulated illumination grating and its inducing photocurrent. Experiments on hydrogenated amorphous silicon prove that an accurate measurement of the diffusion length as well as an identification of ambipolar or nonambipolar diffusion can be acquired by using this technique.
\end{abstract}

\section{INTRODUCTION}

Illumination grating produced by interfering light beams has been widely utilized for investigating the photocarrier diffusion in semiconductors, particularly, in hydrogenated amorphous silicon $(a-\mathrm{Si}: \mathrm{H})$. There exist so far two popular methods which are called the transient grating (TG) technique $e^{1,2}$ and the steady-state photocarrier grating (SSPG) technique. ${ }^{3-5}$ In the former experiment, the interference pattern produces a sinusoidally varying photocarrier concentration, which induces the periodic change in the refractive index and the absorption coefficient of the material. The photocarrier motion via diffusion is then monitored by the time evolution of the light diffracted from these gratings. The SSPG technique does not involve diffraction effects, and instead consists of the steady-state measurement of photocurrent across the concentration grating of which the amplitude is determined by the diffusion length. The transient and the steady-state experiments can both apply to measurements of the diffusion length. A fundamental assumption upon which the experimental results are interpreted is that local space charge neutrality is maintained over the photocarrier grating. Nevertheless, no clear testing criterion for the assumption is involved in the experiments raised above. The existing grating techniques, in this sense, remain less reliable for exact measurements of the diffusion length.

In this article, we investigate theoretically the modulated photocarrier grating (MPG) created by a temporally oscillating interference pattern, and propose an alternative technique that is based on the frequency domain measurement of the phase shift between the illumination and the resulting photocurrent. Expcrimental application to undoped $a-\mathrm{Si}: \mathrm{H}$ demonstrates that the suggested technique is a valuable means to identify local charge neutrality as well as to accurately measure the diffusion length.

\section{THEORY OF THE MODULATED PHOTOCARRIER GRATING TECHNIQUE}

\section{A. Modulated photocurrent due to an oscillating illumination grating}

It may be appropriate to outline the experiment to produce the MPG before going into the mathematical de- scription. As illustrated in Fig. 1, the sample of photoconductor thin film with coplanar contacts is illuminated uniformly by a laser beam of wavelength $\lambda$ and intensity $I_{1}$ at an incident angle $\theta$. A small portion, $I_{2}$, of the beam is split off, passes through an electrooptic modulator for controlling the polarization, and impinges normally on the sample. When these two beams have parallel polarization, an interference occurs and a sinusoidally varying light intensity pattern of the period $\Lambda=\lambda / \sin (\theta)$,

$$
I(x)=I_{1}+I_{2}+2 \gamma_{0} \sqrt{I_{1} I_{2}} \cos (K x),
$$

is formed. Here, $x$ is the direction between the contacts, $K=2 \pi / \Lambda$ the spatial frequency, and $\gamma_{0}\left(0<\gamma_{0}<1\right)$ the interference quality factor. By rotating the plane of polarization of the beam $I_{2}$ by $90^{\circ}$, no interference occurs so that the intensities $I_{1}$ and $I_{2}$ add up linearly and uniformly. Alternating the parallel and perpendicular polarization with a temporal frequency $\omega=2 \pi f$ yields an oscillating illumination grating, which results in a modulation of photocurrent through the sample. The modulated part of photocurrent is measured by a lock-in amplifier.

Note that rotating the plane of the polarization induces no change in the light reflection at the film surface because of the normal incidence. The uniform part of the light intensity in the sample volume is thereby kept constant during the modulation, so that the photocurrent measured is purely due to the illumination grating in the present experiment. This makes an essential difference from the SSPG experiment, in which the less intense beam $I_{2}$ is mechanically chopped, resulting in the incorporation of the temporally varying uniform illumination and the corresponding photocurrent modulation. ${ }^{3}$ Owing to the asymmetrical incidence of the two beams, an optical grating vector is tilted in the sample volume to the $z$ direction perpendicular to the plane of the surface. In the case of interference, therefore, a sinusoidal variation in the light intensity across the film also occurs with the period $\Lambda_{x}$ $=\Lambda \cot \left[\sin ^{-1}(\sin \theta / n) / 2\right]$ where $n$ is the refractive index of the material. It is permissible to ignore the optional nonuniformity so far as the incident angle $\theta$ is small enough for $\Lambda_{z}$ to be much larger compared with the other imposed length scales like the film thickness and the inplane grating period $\Lambda$. 


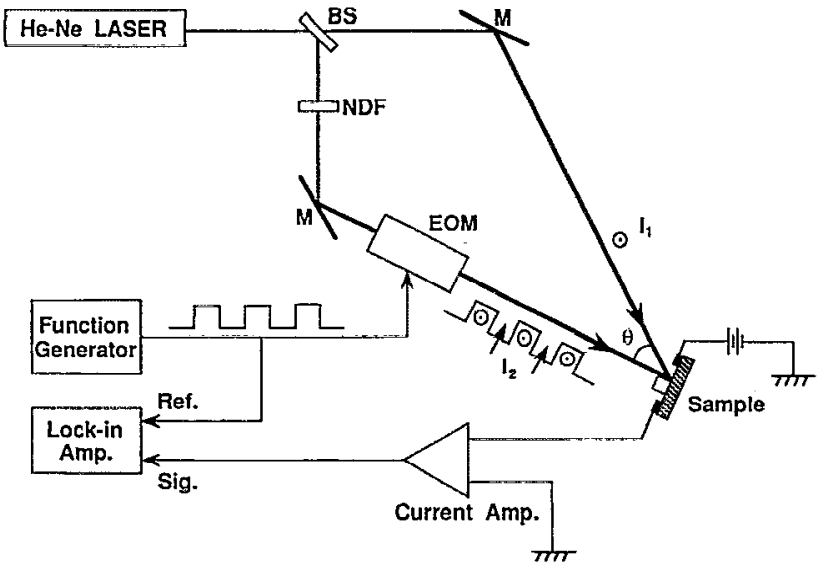

FIG. 1. Experimental setup for the MPG measurement. A linearly polarized laser beam is split by a beam splitter (BS) into two beams 1 and 2 , which are made to coincide on the sample by using the mirrors $(M)$. Beam 2 attenuated by a neutral density filter (NDF) passes through an electro-optic modulator (EOM). These two beams interfere in the case of parallel polarization, while no interference occurs when the plane of polarization of beam 2 is rotated by $90^{\circ}$ by means of the EOM. The parallel and perpendicular polarization is periodically altered. Symbols $\odot$ and $\uparrow$ indicate the electric field vector of the beams. The lock-in amplifier measures the modulated photocurrent due to the polarization modulation.

As described above, the photogeneration rate is comprised of two parts, a spatially uniform part $G_{0}$ and a sinusoidal part $\Delta G$

$$
\begin{aligned}
& G(x, t)=G_{0}+\Delta G(x, t), \\
& \Delta G(x, t)=g \cos (K x)[1+\cos (\omega t)] .
\end{aligned}
$$

$G_{0}$ is constant with time $t$, while $\Delta G$ has a constant term $g$ $\cos (K x)$ and an oscillatory term $g \cos (K x) \cos (\omega t)$. Because $I_{2}$ is chosen to be weaker than $I_{1}$, the nonuniform part of the generation rate is much smaller than the uniform part $\left(\Delta G<G_{0}\right)$, and resulting concentration of photogenerated carriers will also be composed a small nonuniform concentration superimposed on a large uniform concentration. It may be convenient to define the total carrier concentration that consists of the mobile and trapped concentrations. For the small incremental components for electrons $\Delta N$ and for holes $\Delta P$, the linearized transport equations can be represented in a simple fashion $^{4,5}$

$$
\begin{aligned}
\frac{d \Delta N}{d t}= & \Delta G-\frac{\Delta N}{\tau_{n}}-\frac{\Delta P}{\tau_{p}}+\mu_{n} N_{0} \frac{d \Delta E}{d x}+\mu_{n}^{\prime} \frac{d \Delta N}{d x} E_{0} \\
& +D_{n} \frac{d^{2} \Delta N}{d x^{2}}, \\
\frac{d \Delta P}{d t}= & \Delta G-\frac{\Delta N}{\tau_{n}}-\frac{\Delta P}{\tau_{p}}-\mu_{p} P_{0} \frac{d \Delta E}{d x}-\mu_{p}^{\prime} \frac{d \Delta P}{d x} E_{0} \\
& +D_{p} \frac{d^{2} \Delta P}{d x^{2}}, \\
\frac{d \Delta E}{d x}= & \frac{q}{\epsilon}(\Delta P-\Delta N),
\end{aligned}
$$

where $N_{0}$ and $P_{0}$ denote the uniform components of concentrations, and the $E=E_{0}+\Delta E$ the electric field. The parameters appearing in the equation include the following (Refs. 4 and 5): $\mu_{n, p}$ are the drift mobilities, $\mu_{n, p}^{\prime}$ the smallsignal drift mobilities, $D_{n, p}=v \mu_{n, p}^{\prime}$ the effective diffusion constants, $\tau_{n, p}$ the small-signal lifetime, $v$ the thermal voltage, $q$ the elementary electric charge, and $\epsilon$ the dielectric constant. Equations (4)-(6) remain valid in a properly slow modulation case, in which quasithermal equilibrium is settled between small perturbations in mobile and trapped carriers within the period of modulation. The mobile carrier concentrations are then kept at a constant fraction of the total concentration, and the average transport parameters, $\mu_{n, p}^{\prime}, D_{n, p}$, and $\tau_{n, p}$, can be well-defined as timeinvariant quantities.

Solving the equations can be greatly simplified when solution forms

$$
\begin{aligned}
& \Delta N=\operatorname{Re}\left(\sum_{\Omega=-\omega, 0, \omega} n_{\Omega} \exp [i(K x+\Omega t)]\right), \\
& \Delta P=\operatorname{Re}\left(\sum_{\Omega=-\omega, 0, \omega} p_{\Omega} \exp [i(K x+\Omega t)]\right),
\end{aligned}
$$

are applied. It is readily found that the $\Omega=0$ component involved in the summation stands for a solution for the constant term $g \cos (K x)$ in the small-signal photoexcitation $\Delta G$. The $\Omega \neq 0$ components represent waves traveling in the positive and negative $x$ directions, the sum of which forms a standing wave that is a solution for the oscillatory excitation term $g \cos (K x) \cos (\omega t)$. Substituting the above expressions into Eqs. (4)-(6), one obtains two coupled linear equations for the unknown complex amplitudes $n_{\Omega}$ and $p_{\Omega}$

$\overline{n_{\Omega}}\left(\delta+\frac{b c}{b+1}+K^{2} L_{d n}^{2}-i K L_{e n}+i \Omega \tau\right)+\overline{p_{\Omega}}\left(\eta-\frac{b c}{b+1}\right)=1$,

$\overline{n_{\Omega}}\left(\delta-\frac{c}{b+1}\right)+\overline{p_{\Omega}}\left(\eta+\frac{c}{b+1}+K^{2} L_{d p}^{2}+i K L_{e p}+i \Omega \tau\right)=1$,

where $\tau=\left(\tau_{n}^{-1}+\tau_{p}^{-1}\right)^{-1}$ is the common lifetime. $L_{d n, p}$ $=\sqrt{D_{n, p} \tau}$ the diffusion lengths, and $L_{e n, p}=\mu_{n, p}^{\prime} E_{0} \tau$ the drift lengths. The dimensionless constants are defined by $b=\mu_{n}$ / $\mu_{p}, c=\tau / \tau_{d}, \delta=\tau / \tau_{n}$, and $\eta=1-\delta=\tau / \tau_{p} . \tau_{d}$ denotes the dielectric relaxation time $\epsilon / \sigma_{0}$ where $\sigma_{0}=q\left(\mu_{n} N_{0}+\mu_{p} P_{0}\right)$ is the background conductivity. $\overline{n_{\Omega}}$ and $\overline{p_{\Omega}}$ are the amplitudes normalized by $g \tau$ for $\Omega=0$ and by $g \tau / 2$ for $\Omega \neq 0$ and are also dimensionless. These equations for the grating amplitudes are identical with those in the SSPG transport problem $^{4,5}$ except for an additional term $i \Omega \tau$.

We now proceed into the description of modulated photocurrent induced by the MPG. A sum of the conduction current and displacement current should be constant at all $x$. This postulation from the current continuity permits equating the small-signal photocurrent $\Delta J$ produced by the illumination with the cyclic boundary condition $\Delta G(x)=\Delta G(x+\Lambda)$ to its spatial average $\langle\Delta J\rangle$ defined by the integration from $x=0$ to $\Lambda$ divided by $\Lambda .{ }^{5}$ Any contributions from diffusion currents vanish in their spatial av- 
erage process because of the periodicity. An average of an internal electric field induced by the local space charge is also equal to zero. Under the externally applied field $E_{0}$ kept at constant with time, it is fully justified to put the contribution from the displacement current out of the consideration. The small-signal photocurrent is consequently represented only by the drift current components averaged spatially ${ }^{6}$

$$
\Delta J=q\left(\mu_{n}^{\prime}\langle\Delta N \Delta E\rangle+\mu_{p}^{\prime}\langle\Delta P \Delta E\rangle\right) .
$$

As found from Eqs. (7) and (8), the product terms $\langle\Delta N \Delta E\rangle$ and $\langle\Delta P \Delta E\rangle$ involve components that oscillate with frequency $\omega$. The first harmonics are formulated after some mathematical manipulations by

$$
\begin{aligned}
\langle\Delta N \Delta E)\rangle^{\omega} & =\langle\Delta P \Delta E\rangle^{\omega} \\
& =-\frac{q g^{2} \tau^{2}}{4 \epsilon K}[A \cos (\omega t)-B \sin (\omega t)],
\end{aligned}
$$

with the in-phase coefficient $A$ and the quadrature coefficient $B$ defined by

$$
\begin{aligned}
A= & \operatorname{Im}\left(\overline{n_{0}}\right) \operatorname{Re}\left(\overline{p_{-\omega}}+\overline{p_{\omega}}\right)-\operatorname{Re}\left(\overline{n_{0}}\right) \operatorname{Im}\left(\overline{p_{-\omega}}+\overline{p_{\omega}}\right) \\
& +\operatorname{Im}\left(\overline{n_{-\omega}}+\overline{n_{\omega}}\right) \operatorname{Re}\left(\overline{p_{0}}\right)-\operatorname{Re}\left(\overline{n_{-\omega}}+\overline{n_{\omega}}\right) \operatorname{Im}\left(\overline{p_{0}}\right), \\
B= & \operatorname{Im}\left(\overline{n_{0}}\right) \operatorname{Im}\left(\overline{p_{\omega}}-\overline{p_{-\omega}}\right)-\operatorname{Re}\left(\overline{n_{0}}\right) \operatorname{Re}\left(\overline{p_{-\omega}}-\overline{p_{\omega}}\right) \\
& +\operatorname{Re}\left(\overline{n_{-\omega}}-\overline{n_{\omega}}\right) \operatorname{Re}\left(\overline{p_{0}}\right)-\operatorname{Im}\left(\overline{n_{\omega}}-\overline{n_{-\omega}}\right) \operatorname{Im}\left(\overline{p_{0}}\right) .
\end{aligned}
$$

The phase shift $\phi$ between the small-signal photoexcitation and the first harmonic photocurrent is given by

$$
\tan (\phi)=B / A \text {. }
$$

Analysis of the phase shift which is dependent upon the concentration grating amplitudes is a basis of the MPG technique for diffusion length measurements. The detailed discussion concerning how it works will be presented in the next section.

\section{B. Phase shift in the lifetime and relaxation-time regimes}

In this section, we restrict our attention to a weak electric field case in which concentration grating becomes diffusion controlled. A criterion for a negligible field is simply written by $E_{0}<E_{d}$ where $E_{d}=K v$ is the diffusion field. ${ }^{5}$ It is found from the theoretical analysis of a weak field MPG that the in-phase coefficient $A$ as well as the quadrature coefficient $B$ vary linearly with $E_{0}$ so that the small-signal photocurrent shows a linear dependence on $E_{0}$, while $E_{0}$ has no influence on the phase shift $\phi$ determined by the ratio $B / A$. Another important result is that $A$ and $B / \omega \tau$ are both given by functions of $(\omega \tau)^{2}$.

For the sake of simplicity, we also limit the following consideration to a low modulation frequency regime. It should be noticed that this theoretical restriction matches with the application of Eqs. (4)-(6) which are formulated with assuming a slow modulation. A convenient way to deal with the small $\omega \tau$ limit is to use a series expansion in powers of $\omega \tau$. The expansions for $A$ and $B$ are

$$
\begin{aligned}
& A=A_{0}+A_{2}(\omega \tau)^{2}+\cdots, \\
& B=B_{1} \omega \tau+B_{3}(\omega \tau)^{3}+\cdots .
\end{aligned}
$$

Computing the lowest order terms, one obtains the lowfrequency formulas

$$
\begin{aligned}
A=A_{0}=2 & \frac{K L_{e n}\left(c+K^{2} L_{d p}^{2}\right)+K L_{e p}\left(c+K^{2} L_{d n}^{2}\right)}{\left[c\left(1+K^{2} L_{\mathrm{amb}}^{2}\right)+\left(\delta+K^{2} L_{d n}^{2}\right)\left(\eta+K^{2} L_{d p}^{2}\right)-\delta \eta\right]^{2}}, \\
\frac{B}{\omega \tau}=B_{1}= & -\frac{K L_{e n}+K L_{e p}}{\left[c\left(1+K^{2} L_{\mathrm{amb}}^{2}\right)+\left(\delta+K^{2} L_{d n}^{2}\right)\left(\eta+K^{2} L_{d p}^{2}\right)-\delta \eta\right]^{2}}-2\left\{K L _ { e n } \left[\left(\eta+\frac{c}{b+1}+K^{2} L_{d p}^{2}\right)\left(c+K^{2} L_{d p}^{2}\right)\right.\right. \\
& \left.\left.-\left(\eta-\frac{b c}{b+1}\right)\left(c+K^{2} L_{d n}^{2}\right)\right]+K L_{e p}\left[\left(\delta+\frac{b c}{b+1}+K^{2} L_{d n}^{2}\right)\left(c+K^{2} L_{d n}^{2}\right)-\left(\delta-\frac{c}{b+1}\right)\left(c+K^{2} L_{d p}^{2}\right)\right]\right\} / \\
& {\left[c\left(1+K^{2} L_{\mathrm{amb}}^{2}\right)+\left(\delta+K^{2} L_{d n}^{2}\right)\left(\eta+K^{2} L_{d p}^{2}\right)-\delta \eta\right]^{3}, }
\end{aligned}
$$

where $L_{\mathrm{amb}}=\sqrt{D_{\mathrm{am}, \mathrm{b}} \tau}$ is the ambipolar diffusion length and $D_{\text {amb }}=\left(\mu_{n} D_{p}+\mu_{p} D_{n}\right) /\left(\mu_{n}+\mu_{p}\right)$ is the ambipolar diffusion constant. ${ }^{4,5}$ Note that a linear relation stands between $\tan (\phi)=B / A$ and $\omega \tau$ as a direct consequence of the first order approximation. Hence, one may write $\tan (\phi)$ with defining a characteristic time $T=-\tau B_{1} / A_{0}$ as

$$
\tan (\phi)=-\omega T
$$

Experimentally, one can check whether the low-frequency regime discussed here is met by observing the linearity of $\tan (\phi)$ on the frequency $\omega$.

It is instructive to show the reduced expressions of the characteristic time $T$ for two extreme cases, the lifetime regime and the relaxation-time regimes. The lifetime regime refers to the case where the dielectric relaxation time $\tau_{d}$ is much shorter than the carrier lifetime $\tau$. Local space charge neutrality is then maintained despite the different diffusion constants of both types of carriers, and the con- 
centration grating controlled just by the ambipolar diffusion length $L_{\text {amb }}$ results. The ambipolar diffusion has been intensively studied in the SSPG work since it leads to a simple relation between $L_{\mathrm{amb}}$ and the experimental data. ${ }^{3,4}$ Similarly, a direct connection in the MPG can be found between $L_{\text {amb }}$ and the characteristic time $T$. The expression for $T$ is simplified in the $c=\tau / \tau_{d} \rightarrow \infty$ limit to

$$
\tau / T=1+K^{2} L_{\mathrm{amb}}^{2},
$$

indicating that $\tau / T$ is larger than unity for any finite $K$, and exhibits a linear variation against $K^{2}$ with a slope of $1 / L_{\mathrm{amb}}^{2}$. Also interesting to note is that Eq. (19) coincides with the expression for a decay time constant measured in the TG technique: $1 / T=1 / \tau+K^{2} D_{\text {amb }}{ }^{1,2}$ The coincidence obviously arises from equivalent physical processes involved in both the frequency-domain and the time-domain measurements.

On the other hand, an opposite relation stands between $\tau_{d}$ and $\tau$ in the relaxation-time regime. In the $c \rightarrow 0$, limit a somewhat complicated expression

$$
\frac{\tau}{T}=\frac{4\left[\left(\delta+K^{2} L_{d n}^{2}\right)\left(\eta+K^{2} L_{d b}^{2}\right)-\delta \eta\right]}{3\left(1+K^{2} L_{d n}^{2}+K^{2} L_{d p}^{2}\right)-[(\delta / b r)+b r \eta]},
$$

is obtained. The ratio $r$ defined by $b r=\mu_{n}^{\prime} / \mu_{p}^{\prime}$ is a parameter tightly related with the energy distribution of trap states involved. ${ }^{4,5}$ It is evident from the above equation that $\tau / T$ goes towards zero as $K$ bccomes small. The ratio $\tau / T$ at $K=0$ is found directly from Eqs. (16) and (17) to be $(1+1 / 2 c)^{-1}$, which confirms that $\tau / T$ less than unity around $K=0$ is a general trend in the small $c$ case, and gives a clear indication of departing from the lifetime regime in the experimental data.

Whether the above two extreme limits apply to the diffusion of excess carriers under illumination is not clear, a priori, in photoconductive semiconductors such as undoped $a-\mathrm{Si}: \mathrm{H}$, since the conductivity $\sigma_{0}$ as well as the dielectric relaxation time $\tau_{d}=\epsilon / \sigma_{0}$ vary by orders of magnitudes with the illumination level. Therefore, an extended study is needed to discuss the intermediate region between $\tau_{d}<\tau$ and $\tau_{d}>\tau$. The results obtained from examining the case when $\tau_{d}$ is close to $\tau$ are summarized as follows. For $c=1, r=1$, and $\tau_{n}=\tau_{p}(\delta=1 / 2), \tau / T$ can be simply expressed by $\tau / T=(2 / 3)\left(1+K^{2} L_{\text {amb }}^{2}\right)$. In this case, a $\tau / T$ vs $K^{2} L_{\text {amb }}^{2}$ plot shows a straight line which is independent on the drift mobility ratio $b=\mu_{n} / \mu_{p}$. The behavior becomes strongly affected by $b$ unless identical lifetimes are assumed for electrons and holes, as displayed in Fig. 2, where the numerically computed $\tau / T$ is plotted as a function of $K^{2} L_{\text {amb }}^{2}$ for the cases when $\tau_{n}<\tau_{p}(\delta=1)$ and $\tau_{p}<\tau_{n}(\delta$ $=0$ ). In the calculation we assume $r=1$. As found from the figure, a nonlinear relation occurs between $\tau / T$ and

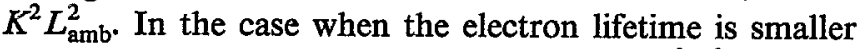
than the hole lifetime, $\tau / T$ decreases with $K^{2} L_{\text {amb }}^{2}$ where $K^{2} L_{\mathrm{amb}}^{2}$ is small, while in the large $K^{2} L_{\text {amb }}^{2}$ region the curve turns upward. An inflection produced in the curve becomes more pronounced for a larger $b$. On the other hand, the $\tau / T$ curve calculated for the smaller hole lifetime relative to the electron lifetime exhibits a completely opposite behavior. $\tau / T$ shows a rapid increase around $K^{2} L_{\text {amb }}^{2}=0$
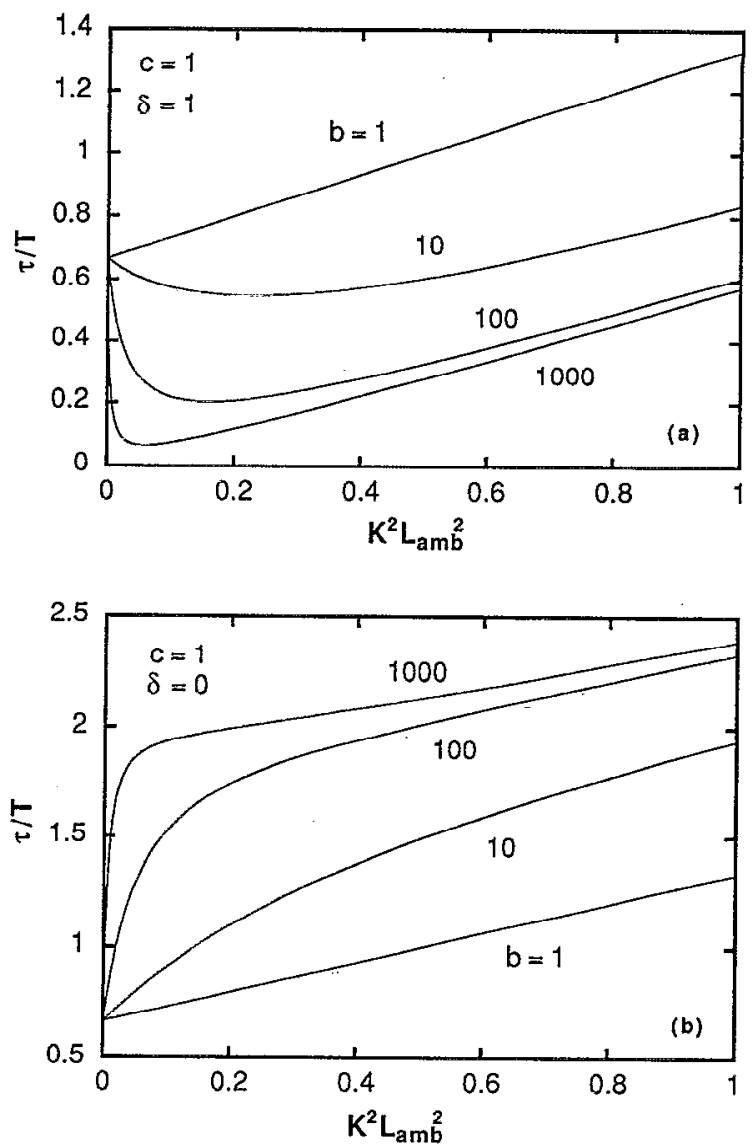

FIG. 2. Theoretical plot of $\tau / T$ vs $K^{2} L_{\text {amb }}^{2}$, for $c=\tau / \tau_{d}=1$ and $\mu_{n}^{\prime} /$ $\mu_{p}^{\prime}=\mu_{n} / \mu_{p}$. The lifetime ratio $\delta=\tau / \tau_{n}$ is set at unity (a) and zero (b). The parameter $b=\mu_{n} / \mu_{p}$ is taken as $1,10,100$, and 1000 .

and then slowly grows with $K^{2} L_{\text {amb }}^{2}$. The striking contrast found between $\delta=0$ and $\delta=1$ cases is preserved even if the $r$ value is varied, and is applicable to an experimental evaluation of the lifetime ratio $\delta$.

\section{DIFFUSION LENGTH MEASUREMENT IN AMORPHOUS SILICON}

\section{A. Determination of the diffusion length from the phase shift analysis}

This section presents the experimental results of the diffusion length measurements on $a-\mathrm{Si}: \mathrm{H}$. Our approach to the accurate determination of diffusion length includes not only the MPG experiment but also the related experiments consisting of the steady-state photoconductivity (SSPC) and the modulated photocurrent (MPC) measurements from which the small-signal mobility-lifetime product $\left(\mu_{n}^{\prime}+\mu_{p}^{\prime}\right) \tau$ and the small-signal lifetime $\tau$ are determined, respectively. The ratio $c$, that is one of the important parameters for applying the MPG theory to the data analysis, can be readily evaluated from the small-signal lifetime and the background conductivity data. The small-signal mobility-lifetime product is dominated by the more mobile or majority carrier while the ambipolar diffusion length is controlled by the less mobile or minority carrier, and thus 
they are correlated by the drift mobility ratio $b$. The relationship is found from their definitions to be

$$
\frac{L_{\mathrm{amb}}^{2}}{\left(\mu_{n}^{\prime}+\mu_{p}^{\prime}\right) \tau v}=\frac{b(r+1)}{(b+1)(b r+1)} .
$$

It is easily understood from the connection that when $\left(\mu_{n}^{\prime}+\mu_{p}^{\prime}\right) \tau$ value is known, $L_{\text {amb }}$ becomes no longer a free parameter in the MPG data analysis. The application of the combined techniques thus aims at the removal of experimental uncertainty as well as the confirmative interpretation of the results of measurements.

A $1-\mu \mathrm{m}$-thick sample of undoped $a-\mathrm{Si}: \mathrm{H}$ deposited on a glass substrate was used in the experiments. The $a-\mathrm{Si}: \mathrm{H}$ film was prepared by if plasma decomposition from a $\left[\mathrm{SiH}_{4}\right] /\left[\mathrm{H}_{2}\right]=1: 9$ gas mixture. The substrate temperature during decomposition was $250^{\circ} \mathrm{C}$. The contacts were the coplanar Al electrodes with a gap of $1 \mathrm{~mm}$ deposited on top of the sample. The sample was exposed for $8 \mathrm{~h}$ to a He-Ne laser $\left(632.8 \mathrm{~nm}, 100 \mathrm{~mW} / \mathrm{cm}^{2}\right)$ so as to minimize the effects of light-induced change in the transport parameters during measurements.

The outline of the MPG experiments is described in Sec. II A. A light from the He-Ne laser was used as the excitation light, and the intensity ratio $I_{2} / I_{1}$ was set at $5 \%$. The spacing of in-plane illumination grating $\Lambda$ was varied from 0.786 to $2.01 \mu \mathrm{m}$ by changing the incident angle $\theta$ from $18.3^{\circ}$ to $53.6^{\circ}$. The vertical grating period $\Lambda_{z}$ is estimated from the experimental condition to be about ten times larger than the period $\Lambda$ as well as the film thickness. Any effects related to the grating along the $z$ direction are therefore considered to be negligibly small, indicating that our MPG theory can be applied successfully. The voltage applied between the contacts was chosen as small as $7 \mathrm{~V}$. From the lower external field $E_{0}(=70 \mathrm{~V} / \mathrm{cm})$ relative to the diffusion field $E_{d}(>780 \mathrm{~V} / \mathrm{cm})$, the weak field condition is confirmed to be fulfilled in the experiment.

The MPC experiment was performed with inserting a polarizer in the path of the polarization-modulated beam $I_{2}$ to prevent two beams from interfering. Since the photogeneration rate is then spatially uniform, the small-signal lifetime $\tau$ is directly determined from the measurement of the phase shift $\phi$, which is simply expressed by $\tan (\phi)$ $=-\omega \tau$ in the low frequency regime..$^{5}$ The small-signal mobility-lifetime product $\left(\mu_{n}^{\prime}+\mu_{p}^{\prime}\right) \tau$ is also easily evaluated from the photocurrent amplitude measured in the frequency region where $\omega \tau<1$ so that the steady-state approximation is practically valid.

Figure 3 summarizes the frequency dependence of $\tan (\phi)$ measured at photogeneration rate $G_{0}=5.61 \times 10^{20}$ $\mathrm{cm}^{-3} \mathrm{~s}^{-1}$. The open and closed circles represent the MPC and MPG experimental results, respectively. An excellent linearity between $\tan (\phi)$ and $\omega$ is read from the figure, indicating the applicability of the low frequency formula given in Sec. II B. A slope of the linear relation for the MPC data yield estimates of the small-signal lifetime $\tau$ at $0.584 \mu \mathrm{s}$. At this illumination level, the background conductivity $\sigma_{0}$ is measured to be $7.21 \times 10^{-6} \mathrm{~S} \mathrm{~cm}^{-1}$, from which the dielectric relaxation time $\tau_{d}$ is calculated to be $0.147 \mu$ s with taking the dielectric constant as 12 for

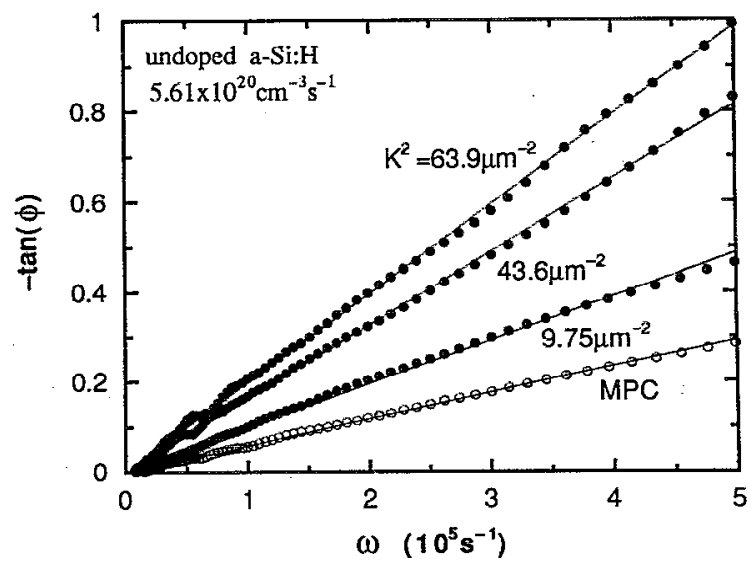

FIG. 3. Tangent of the phase shift $\phi$ of the modulated photocurrent as a function of frequency $\omega$. Open and closed circles represent the MPC and MPG experimental results, respectively. For undoped $a-\mathrm{Si}: \mathrm{H}$ at the background illumination $G_{0}=5.61 \times 10^{20} \mathrm{~cm}^{-3} \mathrm{~s}^{-1}$.

$a$-Si:H. The ratio $c$ is thus determined to be 3.97. The $c$ value on the order of unity implies that nonambipolar diffusion of photocarriers may prevail if there exists a large difference between the drift mobilities for both types of carriers as suggested from our previous estimation.

The characteristic time $T$ determined from the MPG $\tan (\phi)$ spectrum is plotted in the form of the ratio $\tau / T$ against $K^{2}$ in Fig. 4. The nonambipolar diffusion expected above is directly manifested from the ratio $\tau / T$ smaller than unity seen in the figure. It is also found from the figure that $\tau / T$ decreases with $K^{2}$. An important implication from this behavior is, as discussed in Sec. II B, that electron lifetime $\tau_{n}$ is smaller than hole lifetime $\tau_{p}$ so that the common lifetime $\tau$ is dominated by $\tau_{n}$, namely, $\delta=\tau /$ $\tau_{n} \approx 1$. The present conclusion about the lifetime ratio does not contradict with our previous SSPG characterization. ${ }^{5}$ A theoretical curve fitted to the MPG data is plotted by a solid line. In the calculation we use the mobility-lifetime

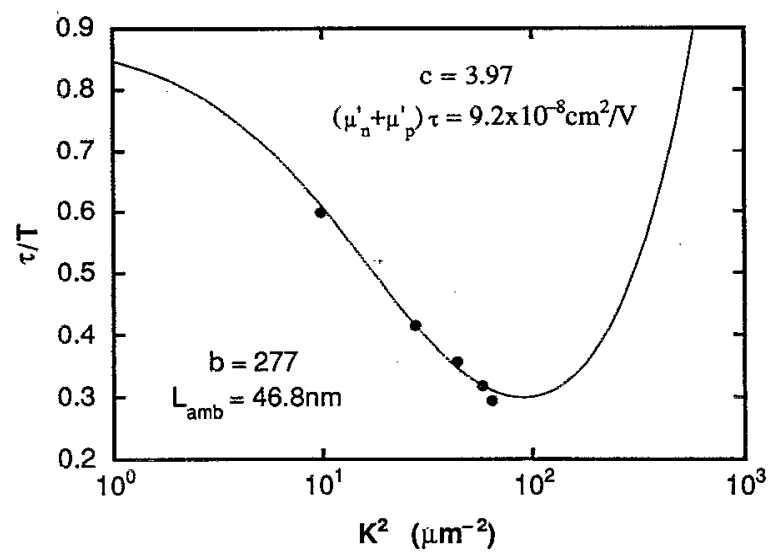

FIG. 4. The ratio $\tau / T$ vs $K^{2}$ of an undoped $a$-Si:H sample. The solid line is a theoretical plot with $c=3.97$ and $\left(\mu_{n}^{\prime}+\mu_{p}^{\prime}\right) \tau=9.2 \times 10^{-8} \mathrm{~cm}^{2} / \mathrm{V} \mathrm{s}$, which are measured at the same illumination level as in the MPG measurements. $b=277$ and $L_{\text {amb }}=46.8 \mathrm{~nm}$ give the best fit. 
product data $\left(\mu_{n}^{\prime}+\mu_{p}^{\prime}\right) \tau=9.2 \times 10^{-8} \mathrm{~cm}^{2} / \mathrm{V}$ and assume that $\delta=1$ and $r=0.6$. The latter assumption is in accordance with the conventional exponential-trap-distribution model in undoped $a$-Si: $\mathrm{H},{ }^{7}$ and has been experimentally verified in the previous work. ${ }^{5}$ The best fit shown in the figure is obtained at $b=277$. Applying Eq. (21), the ambipolar diffusion length is readily determined to be $L_{\text {amb }}$ $=46.8 \mathrm{~nm}$. The ambipolar diffusion length can be deconvoluted into the electron and hole diffusion lengths with using the estimated $b$ value. They are amounted to be $L_{d n}=478$ $\mathrm{nm}$ and $L_{d p}=37.1 \mathrm{~nm}$. Since our study includes the independent measurement of the small-signal lifetime $\tau$, we can also evaluate the small-signal drift mobilities to be $\mu_{n}^{\prime}=0.156 \mathrm{~cm}^{2} / \mathrm{V} \mathrm{s}$ and $\mu_{p}^{\prime}=9.41 \times 10^{-4} \mathrm{~cm}^{2} / \mathrm{V} \mathrm{s}$.

\section{B. Comparison with the result of the steady-state photocarrier grating measurement}

To check the validity of the estimation, we will finally compare it with the results of the SSPG measurement. Details of the SSPG experiments have been given in our previous article. ${ }^{5}$ The background illumination was the same as in the SSPC, MPC, and MPG measurements. The diffusion length determined from the standard SSPG data analysis with assuming ambipolar diffusion is $L_{\text {app }}=102$ $\mathrm{nm}$. As demonstrated in our previous work, ${ }^{5}$ a severe overestimate of diffusion length might result when the ambipolarity is practically lost in the experiment. We therefore need a theoretical translation of the measured $L_{\text {app }}$ into the true diffusion length $L_{\text {amb }}$, without which no firm conclusion of the diffusion length measurement is reached. The procedure for determining $L_{\text {amb }}$ is in principle similar with that discussed in Sec. III A. Using $c$ and $\left(\mu_{n}^{\prime}+\mu_{p}^{\prime}\right) \tau$ values measured independently, a theoretical solution that is consistent with the $L_{\text {app }}$ data is found to be $L_{\text {amb }}=45.1 \mathrm{~nm}$ and $b=298$, both of which are in reasonable agreement with the values evaluated from the MPG measurement. The coincidence lends a trust to two types of experimental approaches to assess the diffusion length.

For $a-\mathrm{Si}: \mathrm{H}$ materials, the most widely used tool for measuring the diffusion length may be the SSPG at present. Although the experimental ease of the SSPG technique makes it attractive for routine characterization, it must be borne in mind that as demonstrated above, the diffusion length $L_{\text {app }}$ is no longer trusted when nonambipolar diffusion takes place in the sample. To experimen- tally identify whether nonambipolar diffusion exists or not as well as whether a calibration of $L_{\text {app }}$ into $L_{\text {amb }}$ is needed or not is therefore of crucial importance for the experimental application. A chief drawback inherent in the SSPG is its deficiency of a simple way for such identification. ${ }^{5}$ The MPG technique developed in this work, in contrast, has a merit of testing ambipolarity directly from its experimental data, offering reliable measurements of the diffusion length.

\section{SUMMARY AND CONCLUSION}

We have studied theoretically the modulated photocurrent induced by a temporally oscillating illumination grating with an emphasis on the relationship between the phase shift and the diffusion length of photocarriers. An analytical formula for the phase shift derived by solving the relevant small-signal transport equations reveals its close connection with the photocarrier grating amplitude controlled by diffusion length. On the basis of the theoretical analysis of the phase shift in all the possible cases including both the ambipolar and nonambipolar diffusion, we have presented a novel and accurate method for testing the ambipolarity as well as for measuring the diffusion length, which we call the MPG technique.

The experimental procedure for evaluating the diffusion length has been demonstrated on an undoped $a-\mathrm{Si}: \mathrm{H}$ sample. In the experiment carried out at an illumination level of the order of $10^{20} \mathrm{~cm}^{-3} \mathrm{~s}^{-1}$, the phase shift analysis yields a clear identification of the nonambipolar diffusion, and estimates of diffusion lengths at $478 \mathrm{~nm}$ for electrons and $37.1 \mathrm{~nm}$ for holes. A reasonable agreement with the SSPG results justifies the MPG measurements, and thus confirms its applicability to the characterization of photoconductive semiconductors.

'S. Komuro, Y. Aoyagi, Y. Segawa, S. Namba, A. Masuyama, H. Okamoto, and Y. Hamakawa, Appl. Phys. Lett. 43, 968 (1983).

${ }^{2}$ K. Hattori, T. Mori, H. Okamoto, and Y. Hamakawa, Appl. Phys. Lett. 51, 1259 (1987).

${ }^{3}$ D. Ritter, K. Weiser, and E. Zeldov, J. Appl. Phys. 62, 4563 (1987).

${ }^{4}$ D. Ritter, E. Zeldov, and K. Weiser, Phys. Rev. B 38, 8296 (1988).

${ }^{5}$ K. Hattori, H. Okamoto, and Y. Hamakawa, Phys. Rev. B 45, 1126 (1992).

${ }^{6}$ In arriving at Eq. (11), we neglect a possible contribution from a second-order correction in perturbation expansion of small-signal conductivity, which has been experimentally confirmed to be negligibly small for a representative sample of undoped $a$-Si:H material (Ref. 5).

${ }^{7}$ T. Tiedje, in Semiconductors and Semimetals, edited by J. Pankove (Academic, New York, 1984), Vol. 21, pt. C, pp. 207-238. 